\title{
Predicting child temperament and behavior from the fetus
}

\author{
JANET A. DIPIETRO, KRISTIN M. VOEGTLINE, HEATHER A. PATER, AND KATHLEEN A. COSTIGAN \\ Johns Hopkins University
}

\begin{abstract}
There remains little debate that the period before birth sets the stage for subsequent development, yet scant evidence exists showing continuity from characteristics of the individual fetus to characteristics of the child. This report examines, in two studies, whether baseline and evoked fetal neurobehavioral functioning are predictive of features of child temperament and behavior as reported by mothers when offspring were between 7 and 14 years old $(M=10.1$ years). Study 1 utilizes data generated from 333 maternal-fetal pairs collected during an undisturbed condition during the second half of gestation in relation to the child temperament dimensions of behavioral inhibition and exuberance. Associations at 32 weeks gestation were detected between all features of fetal neurobehavior and behavioral inhibition. In adjusted models, slower fetal heart rate and less fetal movement were associated with significant unique variance in predicting higher levels of childhood behavioral inhibition. No associations were detected for exuberance. Study 2 focuses on the association of evoked fetal reactivity and recovery to induced maternal arousal with subsequent child behavioral difficulties in a subset of the full sample $(n=130)$. Greater recovery in fetal heart rate following maternal stimulation was predictive of fewer behavioral difficulties and more prosocial behavior in childhood. Results from both studies provide support for gestational origins of core individual differences that portend childhood outcomes with foundational reactivity and regulatory components.
\end{abstract}

Developmental science is predicated on the paradigm that early periods of life are meaningful to later periods. Its core principles concern the development and expression of individual differences and the moderating role of environmental influences across multiple domains that include behavior, physiology, and cognition. Over the past decade or so, the role of the period before birth has received much attention as efforts to identify its influence on postnatal life have intensified. Much of this initial work was generated by epidemiologic studies of health and well-being in adulthood that were retrospectively linked to perinatal circumstances, primarily size at birth (Barker, 1998; O'Brien, Wheeler, \& Barker, 1999). However, interest in the prenatal period among developmentalists is not new. The foundational Fels Longitudinal Study initiated in 1929 included a cohort of pregnant women (Sontag \& Wallace, 1934) and examined the role of the intrauterine and extrauterine environment on the development of the fetus (Sontag, 1941). The invention of technology to better view and monitor the fetus quickly revealed that toward the end of gestation behaviors and other features of developmental function that are routinely measured in the neonate

Prenatal data collection was supported by the Eunice Kennedy Shriver National Institute of Child Health and Human Development (NICHD) award 5R01 HD27592 (to J.A.D); postnatal data collection was supported, in part, by a Johns Hopkins Bloomberg School of Public Health Faculty Initiative Award (to J.A.D.). We thank the dedication and generosity of our study families without whom this work would not be possible.

Address correspondence and reprint requests to: Janet DiPietro, Johns Hopkins Bloomberg School of Public Health, Department of Population, Family and Reproductive Health, 615 N. Wolfe St., W1033, Baltimore, MD 21205; E-mail: jdipiet1@ @hu.edu. and infant neither originate at term gestation nor emerge in response to birth (Als, 1982; Prechtl, 1984). In the mid-1990s, the National Institute of Child Health and Development convened a series of conferences so that neurologists, developmental psychobiologists, developmental psychologists, and obstetricians could share nascent information with the goal of advancing the field of prenatal development and understanding its implications for postnatal life (Krasnegor et al., 1998).

The construct of "fetal programming" has been generated from the most recent wave of work that examines the influences brought to bear on the fetus and features of subsequent development or health. Although we take exception to the use of the term "programming" in application to complex human development outcomes that are multiply determined, there is no doubt that this perspective invigorated interest in the role of the earliest period of development. Nonetheless, the focus of much recent work is not on the fetus per se, but on the maternal and environmental factors that may affect development of one or more fetal organ systems, including the central nervous system. Detection of association with these exposures and subsequent features of health or development in infancy, childhood, or beyond are thus assumed to be attributed to their effect on the fetus and its gestational environment.

Our research program, which began in 1991, has taken a different approach to the study of early origins by developing and applying methodology to measure fetal neurobehavioral development and contemporaneous environmental exposures. The term "neurobehaviors" applies to those features of basic neural functioning that are phenotypic expressions of the processes that underlie the development and expression 
of autonomic and behavioral regulation (Brazelton, 1984). Despite advances in current technologies, the fetus remains relatively inaccessible and fetal neurobehavioral research is limited by what is measurable. The field has generally concentrated on four aspects of fetal function: autonomic (i.e., heart rate and its variation); somatic (i.e., motor activity and patterning); state development and regulation (i.e., coalescence between heart rate and motor activity patterns); and fetal reactivity to stimuli, based on autonomic and somatic responses. All four domains have been shown to develop in predictable ways over gestation (DiPietro et al., 2004; DiPietro, Costigan, \& Voegtline, 2015; DiPietro, Hodgson, Costigan, Hilton, \& Johnson, 1996), including a well-established developmental discontinuity between approximately the 28th and the 32nd gestational week in virtually all aspects of fetal neurodevelopment. For continuous measures, this is expressed as steeper gradient of development up to this gestational age range followed by a slowing of developmental rate through the remaining months of gestation (DiPietro et al., 2015). This is also reflected in specific behaviors and capabilities, including fetal breathing movements (Roodenburg, Wladimiroff, van Es, \& Prechtl, 1991), responsiveness to an external vibrating stimulus (Buss et al., 2009), and habituation (Groome, Gotlieb, Neely, \& Waters, 1993). Patterning of fetal motor activity, heart rate variability, and eye movements undergoes progressive consolidation commencing at about this time, resulting in functional expression as fetal behavioral states corresponding to rudimentary sleep-wake cycles closer to term (Nijhuis et al., 1999; Pillai \& James, 1990). Fetal development is predicated on hierarchical mastery beginning with autonomic control and culminating in interaction with the environment (Als, 1982). Autonomic differentiation both expresses and contributes to developing sympathetic and parasympathetic processes, thereby establishing the basis for reactivity and regulation to endogenous and exogenous stimuli. Both terms are foundational constructs underpinning temperament theory (Goldsmith et al., 1987; Rothbart \& Ahad, 1994).

Remarkable also when considering the fetus is that it serves in an essentially parasitic relationship within another developing human. Figure 1 illustrates our reinvigoration of Als's earlier work (1982), presenting a similar hierarchical structure of fetal neurodevelopment but within a framework of mutual and spiraling engagement between the pregnant woman and fetus. It is difficult, if not impossible, to stimulate the fetus directly without maternal awareness. Instead investigators explicitly rely on inducing maternal physiological activation through the use of experimental manipulations designed to be psychologically challenging or emotionally evocative to generate reactivity in the fetus (Araki et al., 2010; Copher \& Huber, 1967; DiPietro, Ghera, \& Costigan, 2008; Monk et al., 2004). Conversely, spontaneous fetal mo-

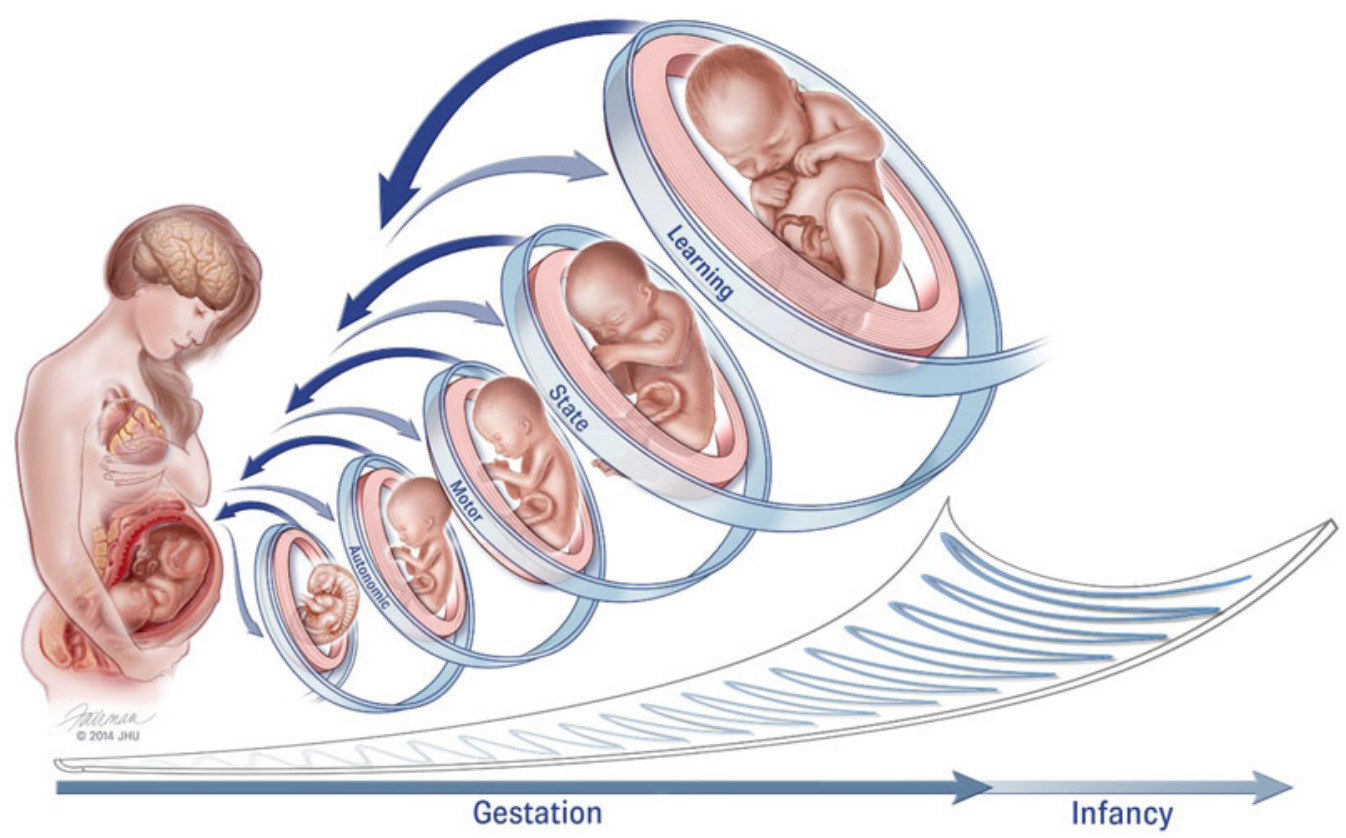

Figure 1. Conceptual model of fetal neurobehavioral development within a framework of bidirectional engagement within the maternal context (reproduced from DiPietro et al., 2015). It is difficult to ascribe specific gestational ages to these domains as development within each progresses from less to more differentiated. For example, while the fetus displays spontaneous movements as early as the 8th week of gestation, movements become progressively more coordinated and consolidated over time. Fetal behavioral states are observed when periods of motor activity are coordinated with patterns of eye movements and variability in heart rate (i.e., autonomic processes), typically commencing near the 32nd gestational week. Fetal learning is predicated on maturation of fetal perception and sensation, stability of fetal behavioral states, and cortical maturation, presumed to occur closer to 36 weeks gestation. Just as in the postnatal period, individual differences in the rate of maturation also contribute variation, in tandem with decreasing canalization as gestation advances and depicted by the "scoop" beneath the figures. 
tor activity inspires maternal physiological reactivity (DiPietro et al., 2006), and stimulating the fetus directly with sound also elicits a maternal response, presumably through evoked fetal behavior (DiPietro et al., 2013).

\section{From Fetus to Child}

The expectation that there are aspects of individuals that endure over time is a given in developmental sciences, yet understanding of which characteristics endure, whether they are the same across individuals, and how to best measure or otherwise detect them has been a challenging endeavor. Various terms, including continuity and stability, have been used (sometimes interchangeably) to describe the preservation of individual differences (i.e., relative or rank ordering of an attribute within a group) across periods of development (Bornstein \& Suess, 2000; Caspi, 1998). Moreover, because the nature of how an underlying attribute is expressed changes as an individual's developmental repertoire expands during maturation, measurement may be homotypic or heterotypic (Putnam, 2011). Heart rate, for example, is a homotypic attribute as it can be measured in similar units over time, but activity level is a heterotypic attribute as the fetus does not locomote, so its expression is necessarily different in the fetus and 3-year-old child. Moreover, both heart rate and motor activity may be viewed as markers of neurologic maturation, particularly when gestational age at the time of measurement is controlled, and thus may be used for heterotypic prediction.

A second consideration in the identification of individual differences involves whether to measure physiological parameters or behaviors during a baseline window of observation in an effort to determine tonic or normative function or perturbing the system to evoke reactivity and recovery, thereby providing some degree of equivalency in context across individuals (Blair, Peters, \& Granger, 2004; Doom \& Gunnar, 2013; Planalp, van Hulle, Gagne, \& Goldsmith, 2017; Porges, Doussard-Roosevelt, Portales, \& Greenspan, 1996). Both approaches have strengths and limitations, but at the core of each is the tenet that individual differences in self-regulation, expressed as both reactivity to stimuli and recovery from that arousal, provide the constitutionally based substrate for broader features of temperament, allowing for refinements over time in conjunction with experience and maturation (Goldsmith et al., 1987; Rothbart \& Derryberry, 1981).

Information on the degree to which characteristics of fetal functioning predict to characteristics of the infant or child is scant. Baseline fetal heart rate and variability are the most stable characteristics during gestation and remain correlated with infant heart rate and variability through at least the first year of life (DiPietro, Costigan, Pressman, \& Doussard-Roosevelt, 2000; Lewis, Wilson, Ban, \& Baumel, 1970), and a small but statistically significant association with 10 -yearold children has been reported (Thomas, Haslum, MacGillivray, \& Golding, 1989). Significant associations with fetal heart rate have been reported between infants with the highest and the lowest reactivity thresholds to novelty (Snidman, Kagan, Riordan, \& Shannon, 1995). Fetal heart rate has been linked with maternally reported infant emotional tone (DiPietro, Hodgson, Costigan, \& Johnson, 1996) and positive reactivity (Werner et al., 2007). Fetal heart rate variability predicts performance on developmental assessments including Bayley psychomotor scores at 18 months (Ratcliffe, Leader, \& Heller, 2002) and both Bayley mental and psychomotor scores, as well as language development and symbolic play, through the 3rd year of life (Bornstein et al., 2002; DiPietro, Bornstein, Hahn, Costigan, \& Achy-Brou, 2007).

With respect to motor activity, more active fetuses tend to become more active neonates (Groome et al., 1999), infants (Degani, Leibovitz, Shapiro, \& Ohel, 2009), and toddlers (DiPietro, Bornstein, et al., 2002), although the latter finding was true only for boys. Fetuses that are more active score higher on behavioral and neurological indicators of motor maturity as neonates (DiPietro et al., 2010) and infants (Richards \& Newbery, 1938). Fetuses that display consistently high levels of motor activity are rated by mothers as more fussy, unadaptable, and unpredictable through 6 months (DiPietro, Hodgson, Costigan, \& Johnson, 1996). Several studies have also documented consistency in aspects of fetal state organization with infant sleep (DiPietro, Costigan, \& Pressman, 2002; DiPietro, Hodgson, Costigan, \& Johnson, 1996; Groome et al., 1997). The longest follow-up of the predictive validity of fetal measures to date reported that near-term fetuses exhibiting more mature transitions between behavioral states were reported by mothers to have better effortful control in late childhood and early adolescence (van den Bergh \& Mulder, 2012) despite the small sample size $(n=25)$. This circumscribed set of findings suggests modest consistency from the prenatal to postnatal periods within specific developmental domains despite the wide contextual variation in which fetal and child measurements are taken.

The literature on fetal reactivity as a predictive construct is even smaller. Two studies have linked fetal responsiveness to maternal physiological arousal, induced through exposure to psychologically challenging or emotionally evocative stimuli, with infant emotion regulation. Greater fetal heart rate responsiveness to a cognitive challenge (i.e., Stroop ColorWord task) presented to pregnant woman predicted greater motor reactivity to a standard novelty paradigm and a trend for greater maternally reported infant negativity at 4 months (Werner et al., 2007). Similarly, fetuses displaying greater heart rate reactivity (as well as motor reactivity) to maternal viewing of a labor and delivery film were more irritable infants in response to the manipulations encountered in a neurodevelopmental exam administered 6 weeks after birth ( $\mathrm{Di}$ Pietro et al., 2008). A recent report notes that lower fetal heart rate variability in response to recorded speech is associated with reduced neurobehavioral maturation in neonates (Figueiredo, Pinto, Pacheco, \& Field, 2017). Greater fetal heart rate reactivity to a vibrating device placed on the maternal abdomen has also been associated with higher maternal ratings 
of fussy/difficultness in early infancy (DiPietro, Hodgson, Costigan, \& Johnson, 1996).

\section{The Current Studies}

This report will extend this limited knowledge base by examining whether tonic and reactive components of fetal functioning are prospectively associated with outcomes in late childhood. Based on the modest but provocative set of findings described above and in concert with the view that individual differences are first manifest in the fetal period, two studies were conducted. The first is based on neurobehavioral and physiological data generated from over 300 maternal-fetal pairs collected during an undisturbed condition at multiple gestational ages; the second utilizes fetal neurobehavioral reactivity and regulation data collected at 24 and 36 weeks from a subset of those cases. We focus on the most conspicuous and measurable facets of fetal neurobehavior: heart rate and motor activity.

Study 1 examines whether fetal neurobehaviors portend child temperament. The temperament dimension of behavioral inhibition, or the tendency to withdraw and behave warily to new people, objects, and situations, was selected because it is perhaps the most extensively studied temperament dimension with well-documented longitudinal stability from early life and underlying physiological correlates (Fox, Snidman, Haas, Degnan, \& Kagan, 2015; Kagan, Reznick, \& Gibbons, 1989; Kagan, Snidman, Kahn, \& Towsley, 2007). Behavioral inhibition is expressed in infancy and early childhood as a characteristic pattern of negative reactivity to novelty; continued behavioral inhibition toward people, both familiar and unfamiliar, is subsequently characterized as shyness. Studies using both laboratory and parental-report measures demonstrate that early behavioral inhibition is predictive of subsequent childhood shyness (Fox, Henderson, Marshall, Nichols, \& Ghera, 2005; Volbrecht \& Goldsmith, 2010), which further confirms temperament stability given that the operational definitions vary with age-dependent developmental capabilities. Although somewhat less commonly investigated, uninhibited behavior has also been investigated as a temperament construct with exuberance as the developmental counterpoint to shyness in later childhood (Dollar, Stifter, \& Buss, 2017; Stifter, Putnam, \& Jahromi, 2008).

Given the limited literature on continuity in temperament from the fetus to child, there is minimal extant data on which to base a directional hypothesis. The two most germane existing findings include a modest link between being classified as a highly reactive infant at 4 months and faster fetal heart rate (Snidman et al., 1995) and detected associations between fetal motor activity and laboratory-assessed behavioral inhibition at age 2 (DiPietro, Bornstein, et al., 2002). In that report, higher fetal motor activity between 24 and 36 weeks gestation was associated with lower behavioral inhibition. Each of these reports is limited by small sample sizes $(n=35$ in both) and relatively short developmental reach. Based on these empirical findings, and the general maturational princi- ples underlying fetal development research, we predict that faster heart rate, greater heart rate variability, and more motor activity will predict less behavioral inhibition in childhood.

Whereas Study 1 is focused on baseline, spontaneous fetal behavior, Study 2 seeks to establish whether fetal reactivity and subsequent regulation elicited by perturbation of the intrauterine environment reveal key attributes of individual differences as they extend to child behavioral difficulties. We rely on evoked maternal autonomic arousal, provided by a standard challenge task, to provide the eliciting stimulus for fetal responsivity. A range of temperament attributes has been linked to perceived behavioral difficulties in children, but here we focus on a general composite of behavioral difficulties as reported by mothers using a structured interview. Again, limited empirical information hinders our ability to generate hypotheses, but we expect that lack of regulation following reactivity in the fetus will extend to indicators of behavioral dysregulation in childhood.

\section{General Method: Overview}

This report is based on a childhood follow-up of 385 children distributed over four cohorts of maternal-fetal pairs that provided prenatal data commencing midway through gestation between June 1997 and July 2007. Women were subsequently surveyed about their child's behavior by phone and mailed questionnaires when children were in late childhood and early adolescence (ages 7 to 14). Although study aims and methods for each prenatal cohort varied, a standard protocol was embedded in each allowing aggregation of baseline fetal data across cohorts; these data form the basis for Study 1. The protocol for the largest of the cohorts included a maternal manipulation implemented to assess fetal reactivity and recovery, providing the Study 2 data. Because the research questions, methods, and dependent and independent measures differ for each study, they are each presented separately.

\section{Study 1: Fetal Neurobehavior and Inhibited/ Uninhibited Temperament}

\section{Participants}

A total of 508 eligible maternal-fetal pairs participated in the fetal studies. Prenatal enrollment for all cohorts was limited to nonsmoking healthy women with singleton pregnancies and without significant preexisting conditions that would jeopardize normal progression of pregnancy at enrollment. Women were self-referred volunteers recruited through local university and hospital publications or referrals from from other participants. Pregnancy dectection was based on early first trimester testing ( $M$ gestational age at pregnancy diagnosis $=4.7$, $S D=1.3$ ), and dating was confirmed by examination and/or ultrasound shortly thereafter ( $M$ gestational age at first prenatal visit $=7.7$ weeks, $S D=1.9$ ). Over time, and after children reached school age, families were contacted through informa- 
tion provided during study enrollment or via public records. The final sample $(n=385)$ includes women who completed the full protocol (interview and questionnaire; $n=333$ ) or the interview alone $(n=53)$. Loss to follow-up was due to inability to locate participants over time $(n=90)$, lack of response to attempted contact $(n=27)$, and declining participation $(n=3)$.

Maternal characteristics, based on data collected prenatally, reflects a population of predominantly well-educated $(M$ maternal education $=16.8$ years $)$, married $(93.8 \%)$, and mature ( $M$ age $=31.6$ years), respondents. Infants were predominantly normally grown $(M$ weight at birth $=3432 \mathrm{~g})$, full-term ( $M$ gestational age at delivery $=39.1$ weeks $)$, and had normal Apgar scores $(M=8.0$ and 8.9 at 1 and $5 \mathrm{~min})$. Half of the offpring were girls (49.5\%), and at follow-up most were either the oldest $(47.9 \%)$ or youngest $(27.6 \%)$ member of the family. Women who provided data about their children were slightly older, $M$ difference $=1.4$ years, $t(506)=3.08, p<.01$, with more years of education, $M$ difference $=1.4$ years, $t(506)=3.08, p<.01$, than those who were eligible but did not participate $(n=120)$. As a result of our long-standing research program in the community, a number of women participated with more than one pregnancy. There were 44 sets of siblings within the total follow-up sample of 385 .

\section{Materials and procedure}

Prenatal data collection overview. The standard fetal monitoring protocol involved a baseline, unperturbed 50-min recording of fetal neurobehavioral and maternal psychophysiological measures. Longitudinal designs were implemented for all cohorts, ranging from two to six visits. A full description of the cohorts, prenatal protocol, and the manner in which the fetal data set was compiled is provided elsewhere (DiPietro et al., 2015). That report, in the form of a monograph, is based solely on documenting prenatal development without any postnatal follow-up. The current study is based on three cohorts with data collection during or near the 24th, 32nd, and 36th weeks of gestation (i.e., cohorts I, III, and VI) and one with only the last two periods (IV). The last two cohorts (VII and VIII) concluded more recently (2013), and those children are not included in this report because they were not of school age at the time of analysis.

Prenatal data collection. Visits were generally conducted in early afternoons (13:00 to 15:00) to control for potential diurnal effects. Fetal position and amniotic fluid volume using a standard index, the amniotic fluid index (AFI), were ascertained through brief ultrasound scans. Women were monitored lying down with head elevated, and tilted slightly to the left to avoid venous compression. Monitoring proceeded for 50 undisturbed minutes. Fetal data were collected from the output port of a Toitu (MT320, Tokyo Japan) fetal actocardiograph, which detects fetal heart rate and motor activity through a single wide array transabdominal Doppler transducer. Data were sampled at $1000 \mathrm{~Hz}$ via an internal analog to digital board using streaming software; analysis proceeded offline using customized software (GESTATE; James Long Company, Caroga Lake NY). Digitized heart rate data were filtered for error rejection based on moving averages using a previously established algorithm; fetal motor activity was calibrated in arbitrary units ranging from 0 to 100 .

Fetal variables were quantified as follows: (a) mean fetal heart rate (FHR), computed in 1-min epochs and averaged over the full recording period; (b) fetal heart rate variability (FHRV), calculated as the standard deviation of FHR values per 1-min epoch, and averaged over the recording; (c) total motor activity (FACTIVE), calculated as the number of bouts identified as each time the actograph signal equaled or exceeded a predetermined threshold (15 a.u.s.), and remained at or above this amplitude for at least 10 contiguous seconds multiplied by the mean duration of each bout(s), yielding the total time spent moving, in seconds, per 50-min recording. This approach for defining actograph-detected movements was validated against ultrasound visualization near the initiation of our research program (DiPietro, Costigan, \& Pressman, 1999). In addition, a composite measure of somatic-cardiac (FM-FHR) coupling was defined as occurring each time a fetal movement bout was accompanied by an excursion in FHR $\geq 5$ beats per minute (bpm) for $\geq 5 \mathrm{~s}$ above the FHR baseline, within $5 \mathrm{~s}$ prior to the movement onset, or $15 \mathrm{~s}$ after it, based on previously developed criteria (Baser, Johnson, \& Paine, 1992; DiPietro, Hodgson, Costigan, Hilton, \& Johnson, 1996). FM-FHR coupling index (COUP-IND) was computed as the number of coupled fetal movements divided by all fetal movements during the observation period. When coupling was detected, the latency between the onset of the fetal movement relative to the onset of the FHR change was calculated in seconds and the mean latency across instances was computed (COUP-LATEN). Because fetal monitoring took place over a 10 -year period, fetal measures were standardized (i.e., $Z$ scored) by cohort to rule out potential signal drift in the fetal monitoring device.

Maternal physiological signals were amplified using a multichannel, electrically isolated, bioamplifier (Model JAD-04; James Long Company, Caroga Lake, NY). An electrocardiogram was recorded from three carbon fiber disposable electrodes in triangulated placement and compiled as maternal heart rate (MHR), computed in 1-min epochs and averaged over the recording. Electrodermal activity was monitored from two silver-silver chloride electrodes with a gelled skin contact area placed on the distal phalanxes of the first and index fingers of the nondominant hand. Skin conductance was measured by administering a constant 0.5 -volt rootmean-square $30 \mathrm{~Hz}$ excitation signal and detecting the current flow and quantified in terms of skin conductance level (SCL), scaled from 0 to 25 microsiemens $(\mu S)$. Data quantification proceeded offline using the PHY General Physiology System and IBI Analysis Systems (James Long Company).

Childhood follow-up. General characteristics about the child (e.g., age and birth order) were collected during the initial phone interview followed by a structured interview about 
child behavior (see Study 2). At the time of phone contact, women were asked to complete and return a temperament questionnaire by mail. $M$ child age at follow-up was 10.1 years with a relatively small standard deviation $(S D=1.4)$, but there was significant range (7.3 years to 14.8). Given that it took a decade to accrue the fetal data, follow-up data collection was a largely unfunded venture that was implemented over time as resources and available personnel allowed. This necessitated the use of two different age-based temperament questionnaires. Women completed either the Temperament in Middle Childhood Questionnaire (TMCQ; version 3.0; Simonds \& Rothbart, 2004); $n=269$, 81\%, or the Early Adolescent Temperament Questionnaire-Revised (EATQ, Parent; Ellis \& Rothbart, 2001); $n=64,19 \%$.

Depending on the dimension, between 5 and 11 individual items scores were summed according to scale instructions, with reverse scoring as appropriate, to yield values for target constructs of shyness, fearfulness, and surgency, which include items consistent with the constructs of behavioral inhibition and exuberance, respectively. Each item is scored on a 5-point scale from 1 (almost always untrue) to 5 (almost always true). Both questionnaires assess the same temperament dimensions of interest using similar items although with some variations based on age-based social and behavioral repertoire. For example, both questionnaires contain very similar items for the construct of shyness (e.g., "Feels shy meeting new people"), but surgency items correspond to age (e.g., "Likes going down high slides or other adventurous activities" is included in the TMCQ while "Wouldn't be afraid to try a risky sport like deep sea diving" is in the EATQ). However, there was an age gradient in the degree to which mothers characterized their children as shy, $r(331)=.12, p<.05$, or surgent, $r(331)=$ $.11, p<.05$, so values were standardized (i.e., $Z$ scored) for the EATQ and the TMCQ separately.

\section{Data analysis}

Hierarchical linear modeling was used to confirm the developmental trends in prenatal measures (Raudenbush \& Byrk,
2002). Exploratory analyses included examining sex differences in dependent (child temperament ratings) and independent (fetal heart rate measures [FHR and FHRV], fetal motor activity [FACTIVE], and their relationship [COUP-IND and COUP-LATEN]), along with maternal context variable associations. Bivariate correlations were performed to establish whether there was sufficient indication of associations between prenatal and child measures to proceed with additional analyses. Detection of significant unadjusted associations resulted in stepwise multiple regressions predicting child temperament measures for each fetal measure, controlling for maternal contextual variables. Entry of the fetal measure at the last step provides a conservative approach by evaluating whether it adds significant unique variance to the maternal measures. In order to ascertain whether shared variance between siblings and mothers contributed to the findings, analyses were rerun excluding the second sibling for each pair. Analysis of variance was used to ascertain nonlinear associations following distribution of the temperament measures into three categories based on quartiles (i.e., lowest, highest, and middle $50 \%$ ).

\section{Results and discussion of Study 1}

Descriptive values for fetal measures of those cases with temperament questionnaire data $(n=333)$ are presented in Table 1. As the data generated by these cohorts reflects a subsample of the data presented previously (DiPietro et al., 2015), mean values are presented only to provide measurement context for the current report. Developmental trends follow those reported on the full sample, which included significant declines in FHR and COUP-LATEN, increases in FHRV and COUP-IND ( $p \mathrm{~s}<.0001)$, and no change in overall motor activity. Data are presented by protocolized gestational period, but actual gestational age at testing, derived from early pregnancy dating, was used in analyses. $M$ gestational ages (weeks) were $24.7(S D=0.7), 32.2(S D=0.7)$, and $36.6(S D=0.6)$ at the three periods studied. Note that the two indicators of coupling between FHR and FM signify

Table 1. Fetal neurobehavioral measures at each gestational period

\begin{tabular}{|c|c|c|c|c|c|c|}
\hline & \multicolumn{6}{|c|}{ Gestational period (weeks) } \\
\hline & \multicolumn{2}{|c|}{24} & \multicolumn{2}{|c|}{32} & \multicolumn{2}{|c|}{36} \\
\hline & $n$ & Mean $(S D)$ & $n$ & Mean $(S D)$ & $n$ & Mean $(S D)$ \\
\hline FHR & 257 & $147.5(5.6)$ & 300 & $142.7(6.8)$ & 287 & $141.8(8.6)$ \\
\hline FHRV & 257 & $6.57(1.52)$ & 300 & $7.92(2.14)$ & 287 & $9.24(2.60)$ \\
\hline FACTIVE & 256 & 953.9 (453.7) & 299 & 847.2 (551.9) & 285 & $946.0(611.0)$ \\
\hline COUP-IND & 257 & $0.19(0.07)$ & 299 & $0.29(0.11)$ & 285 & $0.31(0.10)$ \\
\hline COUP-LATEN & 257 & $5.49(1.71)$ & 299 & $4.18(2.10)$ & 285 & $4.22(1.91)$ \\
\hline
\end{tabular}

Note: FHR, fetal heart rate. FHRV, fetal heart rate variability. FACTIVE, total motor activity. COUP-IND, fetal motor activity-FHR coupling index. COUP-LATEN, in coupling instances mean latency between onset of fetal movement relative to the FHR change. Variation in sample sizes between gestational ages are the results of both missed visits and protocol differences between cohorts. Variation within gestational age are due to occasional data quality difficulties for specific measures. 
different maturational expectations: COUP-IND provides information of the degree to which fetal movements inspire changes in heart rate; COUP-LATEN characterizes how tightly these two events are linked in time.

With respect to temperament ratings, more educated women were less likely to characterize their children as fearful, $r(331)=-.26, p<.001$, but not shy, $r(331)=-.07$, or surgent, $r(331)=.03$. There were no sex differences in maternal ratings. Fear and shyness scale $Z$ scores were significantly correlated, $r(331)=.30, p<.001$. Given that items in each reflect the core construct of behavioral inhibition, and following others (Volbrecht \& Goldsmith, 2010), they were combined (summed) into a composite variable. As expected, surgency was negatively related to fear/shyness, $r(331)=$ $-.39, p<.001$, but analyzed as a separate construct. There was no association between the final $Z$-scored fear/shyness variable and child age at questionnaire administration, $r(331)=-.01$.

Preliminary unadjusted bivariate correlations revealed no significant associations between fetal heart rate, motor activity, or coupling measures and behavioral inhibition (i.e., fear/ shyness composite), or surgency and data collected during the first (24 week) or last (36 week) gestational period. Correlations between fetal measures and behavioral inhibition ranged from $r=.04$ to $r=-.10$ for these periods. Surgency values ranged from $r=-.04$ to $r=.11$; the latter value reflecting the association between surgency and COUP-IND neared, but did not attain significance at 36 weeks $(p=.06)$. As a result, data collected from these periods were not considered further. In contrast, all measured aspects of fetal functioning at the middle gestational period (i.e., 32 weeks) were significantly associated with behaviorally inhibited temperament. These associations were modest but significant: FHR, $r(298)=-.17, p<$ .01 ; FHRV, $r(298)=-.12, p<.05$; FACTIVE, $r(296)=$ $-.16, p<.01$; and COUP-LATEN (but not COUP-IND), $r(298)=-.14, p<.05$. Surgency was not significantly correlated with any fetal measure, $r$ s ranged from .05 to .08 , and was not considered further in continuous models.

In addition to maternal education level ( $M$ presented earlier), prenatal maternal context variables include body mass index at the start of pregnancy $(M=24.0, S D=4.5)$, MHR $(M=86.1 \mathrm{bpm}, S D=9.1)$, and skin conductance $(M=7.0$ $\mu \mathrm{S}, S D=3.6)$ collected at 32 weeks. The 32 -week AFI ( $M$ AFI $=14.3 \mathrm{~cm}, S D=2.9$ ) was also included because of its association with fetal motor activity. Consistent with prior findings (DiPietro et al., 2015), fetuses with more amniotic fluid at 32 weeks moved more than those with less, $r$ (296) $=.19, p<.001$. Associations among maternal contextual variables are presented in Table 2. Most (58\%, 86\%, and 94\%) fetuses had assumed a vertex (i.e., head down) position by 24 , 32 , and 36 weeks, respectively; fetal lie was not associated with fetal motor activity so not included in the models.

Separate regression analyses were constructed for each fetal measure as follows: maternal education was entered in the first step to control for the detected association with behavioral inhibition; intrauterine context variables (MHR, SCL,
Table 2. Correlations among maternal context measures

\begin{tabular}{lrrrr}
\hline \hline & BMI & MHR & SCL & AFI \\
\hline Education (years) & $-.30^{* * *}$ & $-.22^{* *}$ & -.01 & -.03 \\
Body mass index (BMI) $\left.^{\text {Maternal heart rate (MHR) }}\right)^{\mathrm{a}}$ & & $.31^{* *}$ & -.10 & $.12^{*}$ \\
Skin conductance level (SCL) $^{\mathrm{a}}$ & & & .00 & .04 \\
Amniotic fluid index (AFI) & & & & -.01 \\
\hline \hline
\end{tabular}

${ }^{a}$ Maternal heart rate and skin conductance level at 32 weeks gestation. ${ }^{*} p<$ $.05 . * * p<.001$.

and body mass index, along with AFI for FACTIVE) were entered in the next step; and the fetal measure was entered on the final step. Table 3 presents the regression analysis results, and roman numerals indicate the Step 3 results for each separate regression of the individual fetal measures. Maternal education was significantly and negatively associated with temperament ratings while the intrauterine variables, either separately or together, did not add additional variance. Note that Steps 1 and 2 results as presented in Table 3 are based on the equation for FHR. Minor differences of one or two cases with missing data for FACTIVE or COUP-LATEN generated slightly different estimates for Steps 1 and 2 in those equations. With the exception of FHRV, which neared but did not attain significance $(p=.10)$, all fetal measures contributed significant unique variance in the predication of behavioral inhibition at the final step of the equation. Exclusion of siblings that contributed both temperament and 32 week fetal data $(n=32)$ did not alter bivariate or multivariate results (not shown), with the exception that the FHRV association with behavioral inhibition attained significance, $F\left(R^{2}\right.$ change $)=$ $3.77, p=.05$.

A final regression incorporating all of the fetal measures at the final step yielded the following results: multiple $R=.35$, $R^{2} \Delta=.06, F(8,286)=4.92, p<.001$. Individual variables that retained significance in the final equation predicting childhood behavioral inhibition included: maternal education, $t=-4.18, p<.001$; FHR, $t=-3.00, p<.01$; and FACTIVE, $t=-2.12, p<.05$.

Figure 2 provides visual depiction of these results using distribution of behavioral inhibition into quartile-based categories (lowest quartile, $n=79$; highest quartile, $n=73$, and middle half, $n=146$ ). Categorical analyses were conducted to examine potential nonlinear relations not detected by regression analysis and sex differences. Separate 3 (Inhibition Category) $\times 2$ (Sex) analyses of variance did not reveal any sex by fetal measure interactions. Significant post hoc contrasts for behavioral inhibition categories are provided. Note that analyses were conducted using $Z$ scores, but nonstandardized variable values are provided in the figures to provide measurement context. Examination of the mean values suggests that all associations are linear, confirms the correlational and regression findings based on continuous values, and illustrates the particular contrasts between the lowest and highest behavioral inhibition groups. For both cardiac measures 
Table 3. Multiple regression models predicting childhood behavioral inhibition (fear) shyness) from fetal neurobehavioral measures at 32 weeks gestation controlling for maternal context $(n=333)$

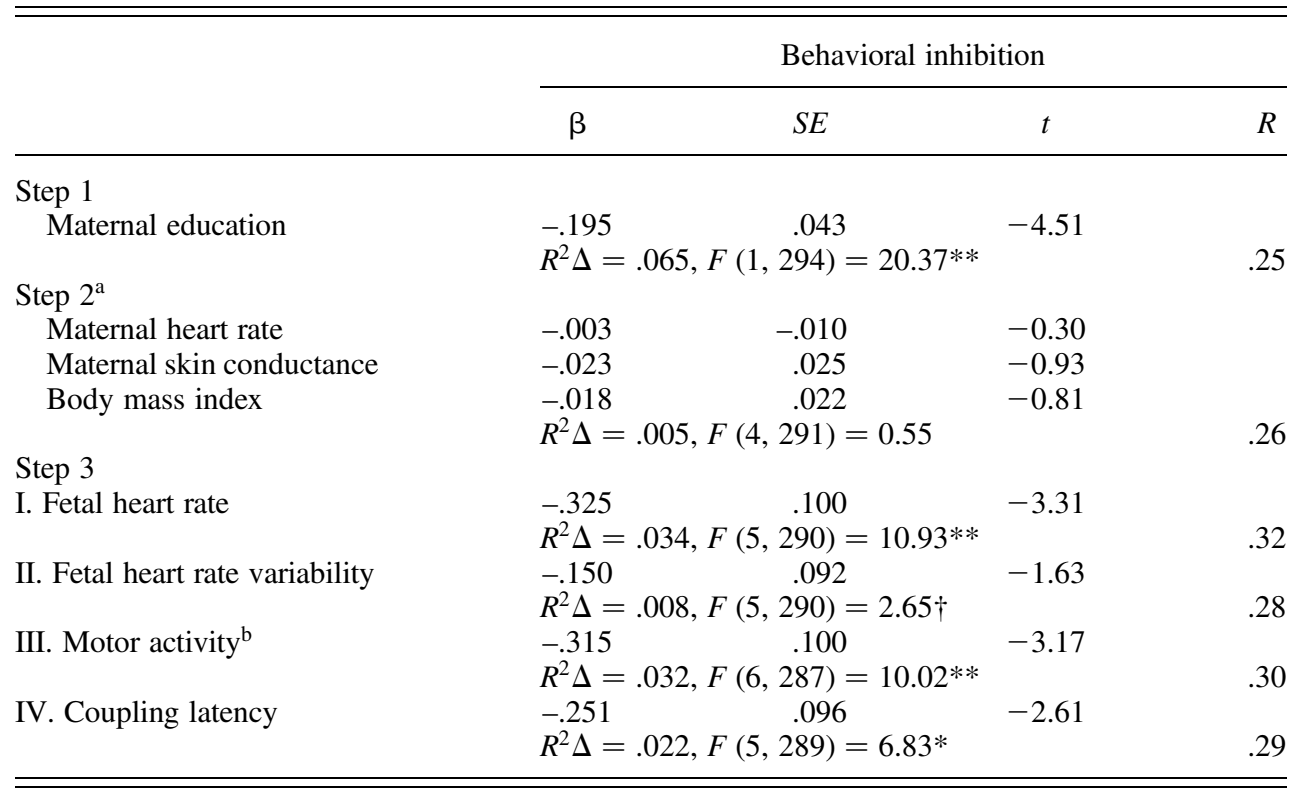

${ }^{a}$ Estimates based on equation for fetal heart rate. ${ }^{\mathrm{b}}$ Amniotic fluid index entered in Step 2 of motor activity equation. $\dagger p=.10 . * p<.01 . * * p<.001$.

(FHR and FHRV) post hoc contrasts revealed significant differences only between the lowest and highest behavioral inhibition groups; for FACTIVE and COUP-LATEN, the highest group differed from both the lowest and middle groups. A sim- ilar approach taken for a categorical surgency variable did not reveal any significant differences (not presented).

Results from Study 1 provide fairly compelling support for the premise that temperamental variation in behavioral inhibi- (a)

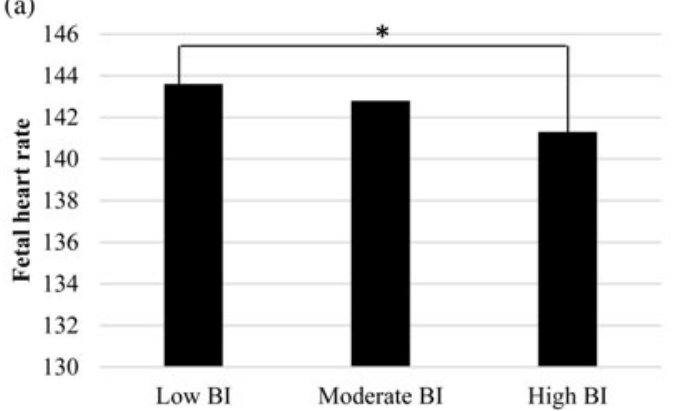

(c)

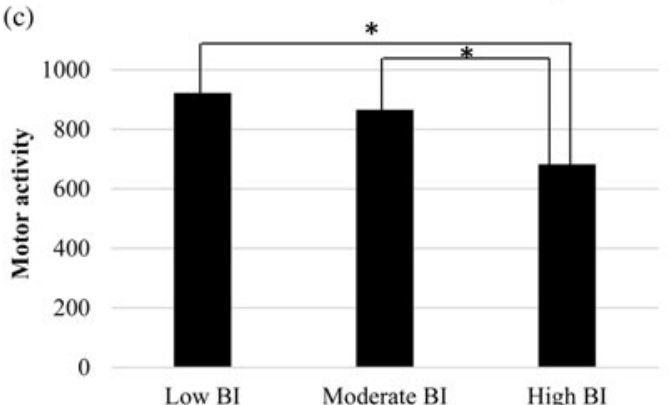

(b)

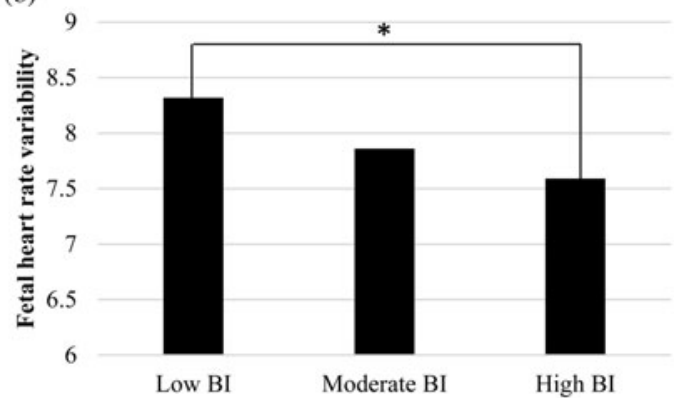

(d)

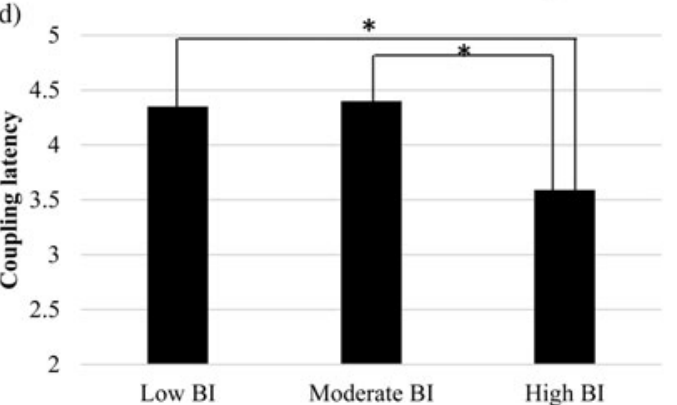

Figure 2. Mean (a) fetal heart rate, (b) fetal heart rate variability, (c) motor activity, and (d) coupling latency by childhood behavioral inhibition. Unadjusted means are presented for visual interpretation, but standardized values were used in analysis. For both cardiac measures (fetal heart rate and fetal heart rate variability), contrasts revealed significant differences only between the lowest and highest groups; for motor activity and coupling latency, the highest group differed from both the lowest and middle groups. $* p<.05$. 
tion, defined here as maternal reports of child fearfulness and shyness, is established before birth. Fetuses exhibiting slower FHR, lower FHRV, and less FM at 32 weeks gestation were described by the mothers as more behaviorally inhibited in late childhood. In addition, the negative association with FMFHR coupling latency implies that when the fetus does move and the movement is sufficient enough to generate perturbation in FHR, the system responds more quickly in fetuses who go on to display behavioral inhibition in childhood. The data analytic method selected is a conservative one because it controls for the relatively large contribution of maternal education on temperament rating, as well as potential maternal physiological influences that could conceivably be related to both the dependent and independent measures. Note that we are assuming that the association between higher maternal education and behavioral inhibition simply reflects a reporting bias, although maternal education may reflect environmental contributors to the expression of child fear/shyness.

Our failure to detect associations with fetal neurobehaviors and maternally reported surgency suggests two related possibilities. The first is that there may be a mismatch between data provided by questionnaires versus the types of measures assessed earlier in childhood in laboratory-based protocols. The second possibility is that, given that surgency and behavioral inhibition scores are negatively correlated, children who score low on behavioral inhibition may be characterized as more "uninhibited" and possess some characteristics of surgent temperament. These individuals occupy the lower end of the continuum reflected in the detected linear associations between behavioral inhibition and fetal measures. Thus, we are hesitant to rule out a prenatal origin for this aspect of temperament based on our assessment method.

The restriction of these results to 32 weeks but not earlier or closer to term may be related to the observed developmental shift in all measured parameters, with the exception of motor activity, that occurs at this gestational age. This period of neural reorganization may maximize detection of individual differences. Before this time, neural immaturity may constrain consolidation of underlying individual differences. As the fetus gets closer to term, physical constraints of the intrauterine environment may limit expression of these differences as factors such as fetal size relative to amniotic fluid, and the intrauterine space can dampen endogenously generated motor activity and resultant changes in heart rate. We will revisit this issue again in the final discussion.

\section{Study 2}

\section{Participants}

Study 2 was based on a subset $(n=130)$ of the larger sample from a cohort that included a fetal reactivity protocol with childhood follow-up data. Maternal (i.e., age, education, and marital status) and fetal (sex, gestational age at delivery, birth weight, and Apgar scores) characteristics were consistent with the larger sample described in Study 1. There were no siblings in this subset.

\section{Procedure}

Fetal and maternal monitoring proceeded as described for Study 1. The longitudinal protocol for one cohort included administration of the Stroop Color-Word test (MacLeod, 1991) following completion of the 50-min undisturbed baseline recordings at 24 and 36 weeks gestation. The task, which requires disassociating word meaning from printed word color under time pressure, evokes a sympathetic response; the version used included pregnancy-specific stimuli as well as standard color words. Details of the protocol have been previously described (DiPietro, Costigan, \& Gurewitsch, 2003). Fetal and maternal data were streamed continuously, and event marking generated three segments: pre-Stroop baseline, Stroop period, and post-Stroop. Child outcome measures were provided during a maternal telephone interview, as described in Study 1.

\section{Measures}

Maternal-fetal reactivity and recovery. To limit the number of analyses, fetal reactivity and regulation to induced maternal arousal was limited to the two principal fetal measures: FHR and FM. Because the experimental segments were too brief for the identification of discrete fetal movement bouts necessary for the total motor activity (and FM-FHR coupling) variables used in Study 1, FM was defined as the summed value of all actograph data points divided by the number of data points per epoch. Mean values were computed by subtracting the Stroop segment from the pre-Stroop baseline (i.e., reactivity) and subtracting the post-Stroop period from the Stroop period (i.e., recovery). MHR and electrodermal (SCL) reactivity to the Stroop were computed for inclusion as contextual variables.

Child behavior at follow-up. Mothers reported on children's behavior using the Strengths and Difficulties Questionnaire (SDQ; Goodman, 2001) administered during a telephone interview. The SDQ is a relatively straightforward appraisal of a child's typical behavioral profile that includes 25 items rated on 3-point scales from not true to certainly true, which are grouped into 5-item subscales: emotional symptoms, conduct problems, hyperactivity, peer problems, and prosocial behavior. The subscales, with the exception of prosocial behavior, are summed to provide a total difficulties score. Psychometric properties of the SDQ have been established in the current sample age range (Goodman \& Goodman, 2009; van Roy, Veenstra, \& Clench-Aas, 2008).

\section{Data analytic plan}

Hierarchical linear models were used to confirm the fetal and maternal response to Stroop as previously presented in the full sample. Pearson correlations were used to evaluate unadjusted bivariate associations between measures of fetal reac- 
tivity/recovery and total difficulties (SDQ-Tot) and prosocial behavior scores (SDQ-Pro), because the latter is not included in the composite score. In addition, given the findings in Study 1, the peer problems score (SDQ-Peer) was also analyzed due to its focus on social interaction. Multivariate regression models were constructed to identify whether fetal responsivity measures were associated with child outcomes using a similar analytic approach as in Study 1. Separate analyses were conducted for each fetal variable at both gestational ages. Categorical analyses based on the level of fetal responsiveness were undertaken using mixed models to examine potential nonlinear associations and interactions with child sex and SDQ-Tot values; contrast estimates tested paired comparisons.

\section{Results and discussion of Study 2}

As described in the original report, the Stroop was effective in eliciting maternal physiological activation consisting of an increase in heart rate and SCL followed by a decrease after the manipulation ceased (DiPietro et al., 2003). Because the current prenatal analysis is based on slightly fewer cases due to exclusion of participants without child follow-up, there is slight variation in the values used in the current analyses, but the overall mean responses remain consistent. The manipulation did not generate a significant mean change in FHR at either gestational age, but did generate suppression of FM at 36 weeks in reaction to the Stroop followed by recovery that returned to or exceeded baseline levels afterward: reactivity, $\beta=-1.65, S E=0.38, t=-4.34, p<.0001$; recovery, $\beta=1.44, S E=0.40, t=3.55, p<.001$. A similar rebound phenomenon was observed at 24 weeks, recovery, $\beta=1.40$, $S E=0.32, t=4.44, p<.0001$, although the initial reaction to the Stroop did not attain significance, reactivity, $\beta=-0.31$, $S E=0.25, t=-1.24, p=.22$.

The original report was focused on main effects for condition and did not consider individual variation. However, Table 4 illustrates the wide individual variation in FHR responsivity. For example, at 36 weeks, FHR reactivity in response to the Stroop ranged from a reduction of $18 \mathrm{bpm}$ to an increase of $22 \mathrm{bpm}$; similar reactivity ranges were found at 24 weeks and also for both recovery values. FM values were also variable although somewhat more constrained. Thus, we examined individual differences in responses throughout this section regardless of central tendency findings.

Scores for SDQ-Tot $(M=6.82, S D=4.65)$, SDQ-Pro $(M$ $=8.84, S D=1.51)$, and SDQ-Peer $(M=1.39, S D=1.45)$ were unrelated to child age at follow-up, $r$ s $(128)=-.10$ to $.06, p s=.26$ to .88 . However, child sex differences were detected for all SDQ outcome variables. Mothers rated boys as having higher SDQ-Tot scores, $t(128)=3.20, p<.01$, lower SDQ-Pro scores, $t(128)=-2.68, p<.01$, and trend-level higher SDQ-Peer scores, $t(128)=1.95, p=.05$. In addition, more highly educated women were less likely to report behavioral problems in their children, SDQ-Tot, $r(128)=-.29$, $p<.001$, including peer problems specifically, SDQ-Peer, $r(128)=-.18, p<.05$, but did not rate their children as more prosocial, SDQ-Pro, $r(128)=.04, p=.65$.

Bivariate associations of fetal response to induced maternal arousal and child behavior. No significant associations emerged between FHR or FM reactivity (i.e., delta from baseline to Stroop periods) and SDQ scores with one exception: a significant association between FM at 24 weeks and SDQPeer, $r(128)=.18, p<.05$. In contrast, there were a number of significant unadjusted associations between fetal recovery (i.e., delta from Stroop to post periods) and SDQ scores. At 24 weeks, FHR recovery was significantly associated with both SDQ-Tot and SDQ-Peer, $r \mathrm{~s}(128)=-.19$, $p \mathrm{~s}<.05$; this relationship was true for all three SDQ measures at 36 weeks, $r s$ (109) range from -.28 to $.23, p s<.05$. That is, larger decreases in FHR following the Stroop were associated with lower SDQ problem scores and higher prosocial scores. Associations with fetal motor activity recovery were limited to 24 weeks, such that larger increases in fetal movement following the Stroop were associated with less SDQ-Pro behavior, $r$ $(119)=.26, p<.01$, and a trend toward more SDQ-Peer problems, $r(119)=-.16, p=.08$.

General linear models of fetal response to induced maternal arousal and child behavior. Tables 5 and 6 provide regression results for 24 and 36 weeks. Because maternal education and child sex were both associated with SDQ ratings, these were

Table 4. Mean response and range of fetal reactivity and recovery scores during and following induced maternal arousal

\begin{tabular}{|c|c|c|c|c|c|c|c|c|}
\hline & \multicolumn{4}{|c|}{24 weeks gestational age } & \multicolumn{4}{|c|}{36 weeks gestational age } \\
\hline & \multicolumn{2}{|c|}{ Reactivity } & \multicolumn{2}{|c|}{ Recovery } & \multicolumn{2}{|c|}{ Reactivity } & \multicolumn{2}{|c|}{ Recovery } \\
\hline & Mean $(S D)$ & Range & Mean $(S D)$ & Range & Mean $(S D)$ & Range & Mean $(S D)$ & Range \\
\hline Fetal heart rate & $0.11(4.5)$ & -11.6 to 17.7 & $0.27(5.3)$ & -21.1 to 16.9 & $0.22(5.7)$ & -22.3 to 18.0 & $-0.35(6.0)$ & -19.1 to 20.4 \\
\hline Fetal movement & $0.31(2.8)$ & -9.9 to 7.4 & $-1.29(3.4)$ & -14.1 to 12.2 & $1.65(4.0)$ & -8.9 to 12.7 & $-1.40(3.5)$ & -11.3 to 5.9 \\
\hline
\end{tabular}

Note: Reactivity values were constructed by subtracting the 2 nd epoch (i.e., Stroop) from the baseline 1st epoch (pre-Stroop baseline); recovery values were constructed by subtracting the 3 rd epoch (i.e., post-Stroop) from the 2 nd epoch (i.e., Stroop). As such, positive change scores indicate a decrease in the Stoop or post-Stroop epoch relative to the prior epoch; negative change scores indicate an increase in the Stroop or post-Stroop epochs relative to the prior epoch. 
Table 5. Multiple regression models: 24-week maternal-fetal responsivity and child behavior

\begin{tabular}{|c|c|c|c|c|c|c|c|c|c|c|c|c|}
\hline & \multicolumn{4}{|c|}{ SDQ total difficulties } & \multicolumn{4}{|c|}{ SDQ peer problems } & \multicolumn{4}{|c|}{ SDQ prosocial skills } \\
\hline & $\beta$ & $S E$ & $t$ & $R$ & $\beta$ & $S E$ & $t$ & $R$ & $\beta$ & $S E$ & $t$ & $R$ \\
\hline \multicolumn{13}{|l|}{ Step 1} \\
\hline Maternal education & -.615 & .174 & $-3.54 * *$ & & -.116 & .057 & $-2.03 *$ & & .026 & .059 & 0.44 & \\
\hline Child sex & -2.46 & .756 & $-3.25 * *$ & & -.479 & .248 & $-1.92 \dagger$ & & .682 & .260 & $2.62 * *$ & \\
\hline & & & & .39 & & & & .24 & & & & .23 \\
\hline \multicolumn{13}{|l|}{ Step 2} \\
\hline MHR reactivity & .020 & .070 & 0.29 & & .011 & .023 & 0.49 & & .045 & .024 & $1.89 \dagger$ & \\
\hline SCL reactivity & .267 & .439 & 0.61 & & .208 & .143 & 1.45 & & .099 & .148 & 0.67 & \\
\hline & & & & .40 & & & & .28 & & & & .29 \\
\hline \multicolumn{13}{|l|}{ Step 3} \\
\hline I. FHR pre & -.009 & .060 & -0.16 & & -.006 & .020 & -0.29 & & -.009 & .021 & -0.42 & \\
\hline FHR reactivity & -.110 & .094 & -1.17 & & .021 & .031 & 0.67 & & -.007 & .033 & -0.22 & \\
\hline FHR recovery & -.166 & .071 & $-2.32 *$ & & -.047 & .023 & $-2.02 *$ & & -.002 & .025 & -0.07 & \\
\hline & & & & .45 & & & & .34 & & & & .29 \\
\hline II.FM pre & -.173 & .135 & -1.28 & & -.016 & .045 & -0.35 & & -.054 & .046 & -1.19 & \\
\hline FM reactivity & .155 & .155 & 1.00 & & .078 & .052 & 1.50 & & .064 & .052 & 1.21 & \\
\hline \multirow[t]{2}{*}{ FM recovery } & -.206 & .119 & $-1.73 \dagger$ & & -.065 & .040 & -1.62 & & .132 & .040 & $3.26 * *$ & \\
\hline & & & & .46 & & & & .35 & & & & .41 \\
\hline
\end{tabular}

Note: SDQ, Strengths and Difficulties Questionnaire. MHR, maternal heart rate. SCL, skin conductance level. Fetal heart rate (FHR) and fetal motor activity (FM) reactivity and recovery values presented for Step 3 reflect separate equations. $\dagger p<.10 .{ }^{*} p<.05 . * * p<.01$.

entered on the first step to control for their effects. Maternal reactivity (MHR and SCL) was entered in the second step, and fetal parameters (reactivity and recovery) entered in the final step. In addition, to control for the law of initial values, fetal baseline values were included in the final step. Maternal education and/or child sex were significantly associated with each SDQ score, multiple $R$ s range from .22 to .39. While maternal physiological reactivity was not associated with SDQ scores at 24 weeks, at 36 weeks there were significant contributions to SDQ-Peer and SDQ-Pro such that women who reacted to the Stroop with greater sympathetic withdrawal (i.e., greater electrodermal decrease) reported their children as having more peer problems and less prosocial behavior. A larger increase in MHR was also associated

Table 6. Multiple regression models: 36-week maternal-fetal responsivity and child behavior

\begin{tabular}{|c|c|c|c|c|c|c|c|c|c|c|c|c|}
\hline & \multicolumn{4}{|c|}{ SDQ total difficulties } & \multicolumn{4}{|c|}{$S D Q$ peer problems } & \multicolumn{4}{|c|}{ SDQ prosocial skills } \\
\hline & $\beta$ & $S E$ & $t$ & $R$ & $\beta$ & $S E$ & $t$ & $R$ & $\beta$ & $S E$ & $t$ & $R$ \\
\hline \multicolumn{13}{|l|}{ Step 1} \\
\hline $\begin{array}{l}\text { Maternal } \\
\text { education } \\
\text { Child sex }\end{array}$ & $\begin{array}{l}-.536 \\
-2.15\end{array}$ & $\begin{array}{l}.197 \\
.823\end{array}$ & $\begin{array}{l}-2.72 * * \\
-2.61 * *\end{array}$ & & $\begin{array}{l}-.111 \\
-.438\end{array}$ & $\begin{array}{l}.065 \\
.270\end{array}$ & $\begin{array}{l}-1.73 \dagger \\
-1.63\end{array}$ & & $\begin{array}{r}-.002 \\
.683\end{array}$ & $\begin{array}{l}.066 \\
.277\end{array}$ & $\begin{array}{r}-0.02 \\
2.47 *\end{array}$ & \\
\hline & & & & .34 & & & & .22 & & & & .23 \\
\hline \multicolumn{13}{|l|}{ Step 2} \\
\hline MHR reactivity & -.002 & .095 & -0.02 & & -.001 & .030 & -0.04 & & .086 & .030 & $2.83 * *$ & \\
\hline SCL reactivity & -.501 & .686 & -0.73 & .35 & .479 & .220 & $2.17 *$ & .30 & -.449 & .220 & $-2.04 *$ & .38 \\
\hline \multicolumn{13}{|l|}{ Step 3} \\
\hline I. FHR pre & .102 & .040 & $2.55^{*}$ & & .034 & .013 & $2.63 * *$ & & -.018 & .013 & -1.38 & \\
\hline FHR reactivity & .012 & .081 & 0.15 & & -.037 & .026 & -1.41 & & .002 & .027 & 0.07 & \\
\hline FHR recovery & -.144 & .068 & $-2.11 *$ & & -.061 & .022 & $-2.79 * *$ & & .045 & .022 & $2.00 *$ & \\
\hline & & & & .47 & & & & .45 & & & & .44 \\
\hline II. FM pre & -.018 & .120 & -0.15 & & .007 & .037 & 0.20 & & .010 & .042 & 0.25 & \\
\hline FM reactivity & -.010 & .143 & -0.07 & & -.055 & .044 & -1.25 & & .012 & .049 & 0.25 & \\
\hline FM recovery & .165 & .133 & 1.25 & & -.021 & .041 & -0.52 & & -.049 & .046 & -1.07 & \\
\hline & & & & .38 & & & & .38 & & & & .41 \\
\hline
\end{tabular}

Note: SDQ, Strengths and Difficulties Questionnaire. MHR, maternal heart rate. SCL, skin conductance level. Fetal heart rate (FHR) and fetal motor activity (FM) reactivity and recovery values presented for Step 3 reflect separate equations. $\dagger p<.10 .{ }^{*} p<.05$. ** $p<.01$. 
with less prosocial behavior. Note that the values provided for Steps 1 and 2 in the tables are based on the equations for FHR; values for FM are similar and follow the same patterns of significance.

With respect to the fetus, at both 24 and 36 weeks, FHR recovery was significantly predictive of SDQ-Tot and SDQPeer, when controlling for all other variables in the equation; at 36 weeks, this association extended to SDQ-Pro as well. That is, fetuses that recovered with greater FHR decline following the Stroop were more likely to be rated as having fewer behavioral difficulties, including peer-related ones, and more prosocial behavior. FM responsivity remained significant only for SDQ-Prosocial skills at 24 weeks, with a trend-level association in the opposite direction for SDQTot. At 36 weeks, FM responsivity was unrelated to child ratings.

Categorical recovery patterns and child behavior. Results presented so far are based on continuous values for change scores; categorical analyses were conducted to better understand the directionality of the fetal recovery response. In addition, review of bivariate correlations between FHR and FM recovery and child measures by fetal sex suggested disparities in the pattern of correlations. Top and bottom quartile groups were constructed based on the direction and magnitude of the change in FHR at 36 weeks from Stroop to post-Stroop periods as follows: activators, FHR increase $\geq+3 \mathrm{bpm}, 24.3 \%, n=27$; suppressors, FHR decrease, $<$ $-3 \mathrm{bpm}, 26.1 \%, n=29$. A similar strategy was applied to FM recovery change scores at 36 weeks: FM increase, $24.8 \%, n=25 ; \mathrm{FM}$ decrease, $24.8 \%, n=25$. Figure 3 depicts SDQ-Tot by FHR/FM recovery groups and sex. Using mixed models, there was a significant FHR Group $\times$ Sex interaction, $F(1,51)=4.84, p<.05$. Pairwise comparisons revealed SDQ-Tot differed for boys versus girls among FHR activators, $t(51)=-3.19, p<.01$. A similar finding, using \pm 3 bpm cutoff values, was replicated at 24 weeks (not shown), Group $\times$ Sex interaction, $F(1,60)=7.56, p<.01, t(60)=$ $-4.16, p<.001$. Although the interaction did not attain significance for FM at 36 weeks, a sex difference was detected among FM suppressors, $t(45)=-2.68, p<.05$.
These results suggest the primacy of fetal poststimulation recovery, as opposed to reactivity, in the prediction from fetal neurobehavioral measures to child outcomes. The SDQ provides a relatively undifferentiated indicator of child tendencies that can be generalized in terms of dysregulation that results in emotional, behavioral, and social disruptions. The results suggest that the degree to which the fetus responds following termination of an environmental challenge, regardless of reactivity to it, provides information about individual differences in neural organization that are manifest in childhood as generalized regulatory problems. For example, at 24 weeks greater rebound in fetal movement following the Stroop is ultimately associated with diminished prosocial behavior, perhaps reflective of a lesser regulatory "brake" that extends to social situations. This seems to be particularly true for boys who displayed a characteristic pattern of post-Stroop activation in heart rate coupled with motor activity suppression. Although the total SDQ score was the principal outcome measure of interest, prosocial ratings were analyzed separately because they were not included in the total score. Findings showing associations with this specific domain of child function, along with its inverse, peer problems, were unexpected. Examination of the items that contribute to those scales suggests they access the degree to which the child gets along with and is helpful to others. This may be an especially particularly salient issue for mothers of children at this age to observe and may be indicative of the broader consequences of behavioral regulation and dysregulation.

\section{General Discussion}

The late-term human fetus and the 10-year-old child inhabit different worlds. The fetus is constrained upside-down, knees near ears, in an obscure, fluid-filled environment; the child is a fully sentient and self-aware being who locomotes freely as she goes about her business. Although both continue on their developmental trajectory, the behavioral repertoires and maturational proficiencies of each are vastly different. Despite this, findings from these two studies indicate that there are attributes that are fundamental to individual differences that can be detected before birth. In Study 1, children who were rated
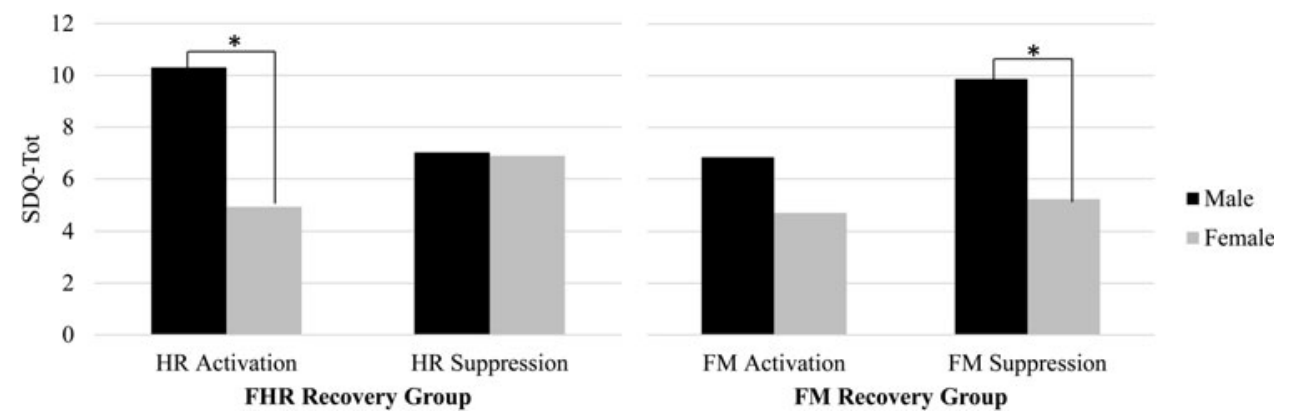

Figure 3. Sex differences in the direction of fetal heart rate (FHR) and fetal motor activity (FM) recovery in relation to Strengths and Difficulties Questionnaire total difficulties at 36 weeks gestation. Male fetuses exhibiting escalation of FHR (at left) or FM suppression (at right) following induced maternal arousal were rated as having more behavioral problems in childhood compared to female fetuses with similar recovery patterns. The same pattern of findings was replicated for FHR at 24 weeks. 
as exhibiting greater fearfulness and shyness were more likely to have slower fetal heart rates and less variability, exhibited less motor activity, and when they did move responded with heart rate reactivity to that motor activity more quickly than fetuses rated as less inhibited in childhood. Taken together, this pattern of findings suggests overall lower autonomic sympathetic and parasympathetic tone interspersed with short latency cardiac reactivity to endogenously generated movements in those fetuses.

The literature on baseline heart rate and behavioral inhibition in early and middle childhood is somewhat mixed, with some reports finding no association while those that do report the opposite of what was detected here, that behaviorally inhibited children display faster heart rate (i.e., generally reported as lower heart period; Fox et al., 2005). The discrepancy may lie in the context in which a fetus and child are measured given that behaviorally inhibited children who are assessed in a laboratory situation may already be displaying sympathetic activation as a result of study participation; the fetus is unaware of being monitored. The detected association between higher spontaneous fetal motor activity and less behavioral inhibition confirmed our earlier report based on laboratory assessment at age 2 (DiPietro, Bornstein, et al., 2002).

In contrast to reliance on tonic (i.e., undisturbed) measurement in Study 1, Study 2 illustrates how a perturbation methodology can be applied to the fetal period to help standardize a window of observation. While conceptually this may be a more appealing approach, it introduces more complexities in interpretation because fetal stimulation is a downstream consequence of maternal reactivity. Although pregnancy is associated with blunted physiological responsiveness, sufficient individual variation is retained (deWeerth \& Buitelaar, 2005). Nonetheless, when a fetus does not display a robust response, it is difficult to ascertain whether this may be the result of constitutional differences in reactivity and regulation of the fetus, the pregnant woman, or both. This may help reconcile, in part, why recovery patterns were more consistently predictive of child outcomes than reactivity ones. Moreover, while we focused on maternal autonomic indicators, there are many unrecorded signals (e.g., changes to the intrauterine acoustic environment) generated by induced maternal arousal that may be transduced to the fetus, making it difficult to fully characterize the maternal response. Despite these challenges, these findings confirm our expectation that individual differences in fetal regulatory function following stimulation are associated with indicators of generalized child dysregulation, which include the social context.

The unique contributions of individual fetal measures to report of child temperament and behavior detected here are significant but not large, accounting for approximately $2 \%$ to $3 \%$ of total explained variance in behavioral inhibition after controlling for the large associations with maternal education on reporting. However, combining all fetal indicators together generated $6 \%$ of explanatory variance, accounted for primarily by fetal heart rate and motor activity, which is comparable to the within-subject stability reported in many longitudinal studies restricted to infancy and childhood. In Study 2, $10 \%$ of unique variance in childhood behavioral difficulties was accounted for by 36 -week fetal heart rate reactivity and recovery combined. Note that baseline (prechallenge) fetal heart rate was also predictive in this instance, such that higher fetal heart rate as well as the postchallenge regulatory response was also associated with total behavior problems in general, and peer problems in particular. This dovetails with the association detected between faster heart rate and less behavioral inhibition in Study 1, particularly as the baseline period used prechallenge was not the same as used in Study 1 .

The other obvious distinction between the fetus and child, in addition to those in posture and capabilities described above, is that one is housed within another individual. While emotional engagement remains in late childhood, the child is no longer physiologically enmeshed. Although baseline maternal physiological indicators have been previously observed to be correlated with fetal heart rate and motor activity within periods of observation, the directionality of this association is not straightforward because the fetus also exerts influence on maternal physiological functioning (DiPietro et al., 2006). In Study 1, neither maternal prenatal autonomic measure contributed to child temperament. In Study 2, the observed associations between both measures of physiological reactivity to challenge and child prosocial behavior at 36 weeks suggests that the reactive maternal component may exert physiological priming of the fetus that translates into childhood behavior. Alternatively, women displaying certain reactivity patterns to challenge (specifically, electrodermal activation coupled with heart rate suppression) are more likely to rate their children positively or provide a child-rearing environment that encourages prosocial development. The latter possibilities presume that maternal reactivity shows stability from pregnancy through the ensuing 10 years, which is not implausible nor mutually exclusive with fetal priming.

Now we turn to what we did not find, associations between fetal measures at the earlier (24 week) and later (36 week) gestational ages and child outcomes in Study 1. This is a not uncommon problem with longitudinal studies with repeated assessments, and interpretation as to why predictive relations are found at one age but not another is not always straightforward. Conversely, had data been collected only at the midpoint of 32 weeks, we might have been tempted to overgeneralize to the entire fetal period. Developmental discontinuities, resulting in temporary reassortment of rank ordering, may play a role. The fetal period is not monolithic and just as there are developmental shifts during the first years of life that are presumed to reflect key periods of neural reorganization (Kagan, 1979; Zeanah, Boris, \& Larrieu, 1997), at least one of these has been established during the prenatal period near 32 weeks gestation, as described and confirmed in the current analysis. Although periods of reorganizational instability may dampen the ability to detect associations, they may also reflect variation in the rate at which individuals mature, thereby distinguishing individuals at a given point in time. In addition, the physical constraints exerted on a developing 
fetus within a limited intrauterine space may diminish the ability to detect associations after this point. However, the preeminence of prediction from 32 weeks was unexpected because our earlier work and that of others has reported associations with early (i.e., first or second year of life) outcomes at 36 weeks or later, and at times, at earlier points. It is possible that the less differentiated behavioral repertoire of younger children makes it easier to identify links to neurobehaviors.

The other possibility is that similar associations exist at the other gestational ages, but based on the considerable signalto-noise ratio in the fetal data, we were unable to detect them in this sample. That is, given the circumstantial differences between the fetus and the child, variation in the intrauterine context during gestation, and the degree of measurement error inherent to collecting data from a study participant that cannot be directly viewed or handled, true shared variance between fetal indicators and child outcomes may be obscured. Stability in fetal cardiac measures and motor activity has been detected as early as 20 or 24 weeks and persists through term (DiPietro et al., 2007, 2015), making the inability to detect associations with undisturbed functioning at the earlier or later periods more puzzling. In Study 2, although significant associations were not consistently found for both gestational ages studied, significant findings were detected at the earlier (24 weeks) and later (36 weeks) periods. This includes replication of the association between recovery of fetal heart rate following induced maternal arousal in relation to total behavioral difficulties and peer difficulties at both 24 and 36 weeks, along with the significant sex difference. While this may reveal a benefit of standardizing the window of observation through perturbation, we have no ready explanation for the lack of findings at these gestational ages in Study 1.

The clear methodological limitation of this report lies in its reliance on maternal report for both studies as opposed to measuring child temperament or behavior in a laboratory setting. While this approach tends to be the rule rather than the exception in studies predicting outcomes from the prenatal period, until the data can be confirmed by observational methods, we regard the findings as provocative but preliminary. However, given the difficulty in collecting data on large prenatal cohorts and the ensuing interim until they reach childhood, we are not optimistic that such an opportunity of equivalent follow-up duration or sample size will arise in the near future. This report was able to leverage the large extant fetal data set, generated by collapsing across multiple cohorts, to extend the reach of prediction from the prenatal

\section{References}

Als, H. (1982). Toward a synactive theory of development: Promise for the assessment and support of infant individuality. Infant Mental Health Journal, 3, 229-243.

Araki, M., Nishitani, S., Ushimaru, K., Masuzaki, H., Oishi, K., \& Shinohara, K. (2010). Fetal response to induced maternal emotions. Journal of Physiological Sciences, 60, 213-202.

Barker, D. (1998). In utero programming of chronic disease. Clinical Sciences, 2, 115-128. period to late childhood and early adolescence in over 300 maternal-fetal pairs. In addition to the benefit conferred by the sample size, validity of the current findings is bolstered by the fact that women were unaware of the independent measures of fetal functioning, thereby precluding reporting biases on that basis. This includes fetal motor activity, as even if women could accurately recall how vigorously a child moved before birth 10 years later, felt fetal movements constitute only a small proportion of fetal movements. Without a systematic source of bias, reliance on maternal report in this context would tend toward a Type II error of interpretation, such that it introduces measurement error that would diminish the ability to detect significant associations that exist.

The findings presented here represent the most comprehensive evaluation to date of the fetal origins of childhood temperament and behavioral outcomes. Change and constancy in human development has been a long-running theme in developmental science (Bornstein \& Suess, 2000; Kagan, 1979; Putnam, 2011; Rothbart, Ahadi, \& Evans, 2000), and here we show that over fairly protracted differences in time and place, core features of individual differences, are preserved. The substrate of individual autonomic and behavioral variation in reactivity and subsequent regulation undergirds the current focus on behavioral inhibition (i.e., fear and shyness) and behavioral regulation or dysregulation (i.e., problem behaviors). The model presented in Figure 1 includes the construct of canalization, indicated by the widening scoop at bottom, connoting the decrease in constraints imposed by species-typical processes earlier in development toward expression of individual differences as development progresses (McCall, 1981). The period of measurement in this report reflects a relatively canalized period of development, yet sufficient individual variation exists to detect longer term extensions. While the current findings confirm the supposition of the constitutionality of these core dimensions, they do not reveal its source. Contributors to individual differences in the fetus likely include genetic influences, those introduced to the intrauterine environment by endogenous and exogenous maternal factors, and more diffuse environmental influences that may affect the fetus directly or though epigenetic alterations. The degree and manner in which these intrauterine and environmental influences displace an individual's developmental trajectory from normative levels of reactivity and regulation toward dysregulational ones continues to be a key area of developmental inquiry.

Baser, I., Johnson, T. R. B., \& Paine, L. L. (1992). Coupling of fetal movement and fetal heart rate accelerations as an indicator of fetal health. Obstetrics and Gynecology, 80, 62-66.

Blair, C., Peters, R., \& Granger, D. (2004). Physiological and neuropsychological correlates of approach/withdrawal tendencies in preschool: Further examination of the Behavioral Inhibition System/Behavioral Activation System scales for young children. Developmental Psychobiology, 45, 113-124. 
Bornstein, M. H., DiPietro, J. A., Hahn, C. S., Painter, K., Haynes, O. M., \& Costigan, K. A. (2002). Prenatal cardiac function and postnatal cognitive development: An exploratory study. Infancy, 3, 475-494.

Bornstein, M. H., \& Suess, P. E. (2000). Child and mother cardiac vagal tone: Continuity, stability, and concordance across the first 5 years. Developmental Psychology, 36, 54-65.

Brazelton, T. B. (1984). Neonatal Behavioral Assessment Scale (2nd ed). Philadelphia: Lippincott.

Buss, C., Davis, E., Class, Q., Gierczak, M., Pattillo, C., Glynn, L., \& Sandman, C. (2009). Maturation of the human fetal startle response: Evidence for sex-specific maturation of the human fetus. Early Human Development, 85, 633-638.

Caspi, A. (1998). Personality development across the life course. In W. Damon, R. Lerner, \& N. Eisenberg (Eds.), Handbook of child psychology: Vol. 3. Social, emotional and personality development (pp. 311-388). Hoboken, NJ: Wiley.

Copher, D. E., \& Huber, C. (1967). Heart rate response of the human fetus to induced maternal hypoxia. American Journal of Obstetrics and Gynecology, 98, 320-335.

Degani, S., Leibovitz, Z., Shapiro, I., \& Ohel, G. (2009). Twins' temperament: Early prenatal sonographic assessment and postnatal correlation. Journal of Perinatology, 29, 337-342.

deWeerth, C., \& Buitelaar, J. K. (2005). Physiological stress reactivity in human pregnancy-A review. Neuroscience and Biobehavioral Review, 29, 295-312.

DiPietro, J. A., Bornstein, M. H., Costigan, K. A., Pressman, E. K., Hahn, C. S., Painter, K., . . Yi, L. J. (2002). What does fetal movement predict about behavior during the first two years of life? Developmental Psychobiology, 40, 358-371.

DiPietro, J. A., Bornstein, M. H., Hahn, C. S., Costigan, K. A., \& Achy-Brou, A. (2007). Fetal heart rate and variability: Stability and prediction to developmental outcomes in early childhood. Child Development, 78, 1788-1798.

DiPietro, J. A., Caulfield, L. E., Costigan, K. A., Merialdi, M., Nguyen, R. H. N., Zavaleta, N., \& Gurewitsch, E. (2004). Fetal neurobehavioral development: A tale of two cities. Developmental Psychology, 40, 445-456.

DiPietro, J. A., Caulfield, L. E., Irizarry, R. A., Chen, P., Merialdi, M., \& Zavaleta, N. (2006). Prenatal development of intrafetal and maternal-fetal synchrony. Behavioral Neuroscience, 120, 687-701.

DiPietro, J. A., Costigan, K., \& Gurewitsch, E. (2003). Fetal response to induced maternal stress. Early Human Development, 74, 125-138.

DiPietro, J. A., Costigan, K. A., \& Pressman, E. K. (1999). Fetal movement detection: Comparison of the Toitu actograph with ultrasound from 20 weeks gestation. Journal of Maternal-Fetal Medicine, 8, 237-242.

DiPietro, J. A., Costigan, K. A., \& Pressman, E. K. (2002). Fetal state concordance predicts infant state regulation. Early Human Development, 68, 1-13.

DiPietro, J. A., Costigan, K. A., Pressman, E. K., \& Doussard-Roosevelt, J. (2000). Antenatal origins of individual differences in heart rate. Developmental Psychobiology, 37, 221-228.

DiPietro, J. A., Costigan, K., \& Voegtline, K. (2015). Studies in fetal behavior: Revisited, renewed, and reimagined. Monographs of the Society for Research in Child Development, 80(Serial no. 318), 1-151.

DiPietro, J. A., Ghera, M. M., \& Costigan, K. A. (2008). Prenatal origins of temperamental reactivity in infancy. Early Human Development, 84, 569-575.

DiPietro, J. A., Hodgson, D. M., Costigan, K. A., Hilton, S. C., \& Johnson, T. R. B. (1996). Development of fetal movement-fetal heart rate coupling from 20 weeks through term. Early Human Development, 44, 139-151.

DiPietro, J. A., Hodgson, D. M., Costigan, K. A., Hilton, S. C., \& Johnson, T. R. B. (1996). Fetal neurobehavioral development. Child Development, 67, 2553-2567.

DiPietro, J. A., Hodgson, D. M., Costigan, K. A., \& Johnson, T. R. B. (1996). Fetal antecedents of infant temperament. Child Development, 67, 25682583.

DiPietro, J. A., Kivlighan, K. T., Costigan, K. A., Rubin, S. E., Shiffler, D. E., Henderson, J., \& Pillion, J. P. (2010). Prenatal antecedents of newborn neurological maturation. Child Development, 81, 115-130.

DiPietro, J. A., Voegtline, K. M., Costigan, K. A., Aguirre, F., Kivlighan, K., \& Chen, P. (2013). Physiological reactivity of pregnant women to evoked fetal startle. Journal of Psychosomatic Research, 75, 321-326.

Dollar, J., Stifter, C. A., \& Buss, K. (2017). Exuberant and inhibited children: Person-centered profiles and links to social adjustment. Developmental Psychology, 53, 1222-1229.

Doom, J., \& Gunnar, M. (2013). Stress physiology and developmental psychopathology. Development and Psychopathology, 25, 1359-1373.
Ellis, L., \& Rothbart, M. (2001). Revision of the Early Adolescent Temperament Questionnaire (EATQ-R). Paper presented at the Biennial Meeting of the Society for Research in Child Development, Minneapolis, MN.

Figueiredo, B., Pinto, T., Pacheco, A., \& Field, T. (2017). Fetal heart rate variability mediates prenatal depression effects on neonatal neurobehavioral maturity. Biological Psychology, 123, 294-301.

Fox, N. A., Henderson, H. A., Marshall, P., Nichols, K., \& Ghera, M. (2005). Behavioral inhibition: Linking biology and behavior within a developmental framework. Annual Reviews of Psychology, 56, 235-262.

Fox, N. A., Snidman, N., Haas, S., Degnan, K., \& Kagan, J. (2015). The relation between reactivity at 4 months and behavioral inhibition in the second year: Replication across three independent samples. Infancy, 20, 98-114.

Goldsmith, H., Buss, A., Plomin, R., Rothbart, M., Thomas, A., Chess, S., . . McCall, R. (1987). Roundtable: What is temperament? Four approaches. Child Development, 58, 505-529.

Goodman, A., \& Goodman, R. (2009). Strengths and Difficulties Questionnaire as a dimensional measure of child mental health. Journal of the American Academy of Child \& Adolescent Psychiatry, 48, 400-403.

Goodman, R. (2001). Psychometric properties of the Strengths and Difficulties Questionnaire. Journal of the American Academy of Child \& Adolescent Psychiatry, 40, 1337-1345.

Groome, L., Singh, K., Bentz, L., Holland, S., Atterbury, J., Swiber, M., \& Trimm, R. (1997). Temporal stability in the distribution of behavioral states for individual human fetuses. Early Human Development, 48, 187-197.

Groome, L., Swiber, M., Holland, S., Bentz, L., Atterbury, J., \& Trimm, R. (1999). Spontaneous motor activity in the perinatal infant before and after birth: Stability in individual differences. Developmental Psychobiology, $35,15-24$.

Groome, L. J., Gotlieb, S. J., Neely, C. L., \& Waters, M. D. (1993). Developmental trends in fetal habituation to vibroacoustic stimulation. American Journal of Perinatology, 10, 46-49.

Kagan, J. (1979). The form of early development: Continuity and discontinuity in emergent competencies. Archives of General Psychiatry, 36, 10471054.

Kagan, J., Reznick, J., \& Gibbons, J. (1989). Inhibited and uninhibited types of children. Child Development, 60, 838-845.

Kagan, J., Snidman, N., Kahn, V., \& Towsley, S. (2007). The preservation of two infant temperaments into adolescence. Monographs of the Society for Research in Child Development, 72(Serial No. 287), 1-95.

Krasnegor, N., Fifer, W., Maulik, D., McNellis, D., Romero, R., \& Smotherman, W. (1998). Fetal behavioral development: A transdisciplinary perspective for assessing fetal well-being and predicting outcome. Prenatal and Neonatal Medicine, 3, 185-190.

Lewis, M., Wilson, C., Ban, P., \& Baumel, M. (1970). An exploratory study of resting cardiac rate and variability from the last trimester of prenatal life through the first year of postnatal life. Child Development, 41, 799-811.

MacLeod, C. (1991). Half a century of research on the Stroop effect: An integrative review. Psychological Bulletin, 109, 163-203.

McCall, R. B. (1981). Nature-nuture and the two realms of development: A proposed integration with respect to mental development. Child Development, 52, 1-12.

Monk, C., Sloan, R. P., Myers, M. M., Ellman, L., Werner, E., Jeon, J., . . Fifer, W. P. (2004). Fetal heart rate reactivity differs by women's psychiatric status: An early marker for developmental risk? Journal of the American Academy of Child \& Adolescent Psychiatry, 43, 283-290.

Nijhuis, I. J. M., ten Hof, J., Nijhuis, J. G., Mulder, E. J. H., Narayan, H., Taylor, D. J., \& Visser, G. H. A. (1999). Temporal organisation of fetal behavior from 24 weeks gestation onwards in normal and complicated pregnancies. Developmental Psychobiology, 34, 257-268.

O'Brien, P., Wheeler, T., \& Barker, D. (Eds.). (1999). Fetal programming. Influences on development and disease in later life. London: RCOG Press.

Pillai, M., \& James, D. (1990). The development of fetal heart rate patterns during normal pregnancy. Obstetrics \& Gynecology, 76, 812-816.

Planalp, E., van Hulle, C., Gagne, J., \& Goldsmith, H. (2017). The infant version of the Laboratory Temperament Assessment Battery (Lab-TAB): Measurment properties and implications for concepts of temperament. Frontiers in Psychology, 8, 846.

Porges, S. W., Doussard-Roosevelt, J. A., Portales, A. L., \& Greenspan, S. I (1996). Infant regulation of the vagal "brake" predicts child behavioral problems: A psychobiological model of social behavior. Developmental Psychobiology, 29, 697-712.

Prechtl, H. (1984). Continuity and change in early neural development. In H. Prechtl (Ed.), Clinics in developmental medicine: Vol. 94. Continuity 
in neural functions from prenatal to postnatal life (pp. 1-15). Philadelphia, PA: Lippincott.

Putnam, S. P. (2011). Stability and instability of childhood traits: Implications for personality development of animals. Developmental Psychobiology, 53, 510-520.

Ratcliffe, S., Leader, L., \& Heller, G. (2002). Functional data analysis with application to periodically stimulated foetal heart rate data: I. Functional regression. Statistics in Medicine, 21, 1103-1114.

Raudenbush, S. W., \& Byrk, A. S. (2002). Hierachical linear models: Applications and data analysis methods (2nd ed.). Thousand Oaks, CA: Sage.

Richards, T., \& Newbery, H. (1938). Studies in fetal behavior: III. Can performance on test items at six months postnatally be predicted on the basis of fetal activity? Child Development, 9, 79-86.

Roodenburg, P. J., Wladimiroff, J. W., van Es, A., \& Prechtl, H. F. R. (1991). Classification and quantitative aspects of fetal movements during the second half of normal pregnancy. Early Human Development, 25, 19-35.

Rothbart, M., Ahadi, S., \& Evans, D. (2000). Temperament and personality: Origins and outcomes. Journal of Personality and Social Psychology, 78, $122-135$.

Rothbart, M., \& Derryberry, D. (1981). Development of individual differences in temperament. In H. Lamb \& A. Brown (Eds.), Advances in developmental psychology (Vol. 1). Hillsdale, NJ: Erlbaum.

Rothbart, M. K., \& Ahad, S. A. (1994). Temperament and the development of personality. Journal of Abnormal Psychology, 103, 55-66.

Simonds, J., \& Rothbart, M. (2004). The Temperament in Middle Childhood Questionnaire (TMCQ): A computerized self-report measure of temperament for ages 7 to 10. Paper presented at the Occasional Temperament Conference, Athens, GA.
Snidman, N., Kagan, J., Riordan, L., \& Shannon, D. (1995). Cardiac function and behavioral reactivity during infancy. Psychophysiology, 32, 199-207.

Sontag, L. W. (1941). The significance of fetal environmental differences. American Journal of Obstetrics and Gynecology, 42, 996-1003.

Sontag, L. W., \& Wallace, R. F. (1934). Preliminary report of the Fels Fund. American Journal of Diseases of Children, 48, 1050-1057.

Stifter, C. A., Putnam, S., \& Jahromi, L. (2008). Exuberant and inhibited toddlers: Stability of temperament and risk for problem behavior. Development and Psychopathology, 20, 401-421.

Thomas, P. W., Haslum, M. N., MacGillivray, I., \& Golding, M. J. (1989). Does fetal heart rate predict subsequent heart rate in childhood? Early Human Development, 19, 147-152.

van den Bergh, B. R., \& Mulder, E. J. (2012). Fetal sleep organization: A biological precursor of self-regulation in childhood and adolescence? Biological Psychology, 89, 584-590.

van Roy, B., Veenstra, M., \& Clench-Aas, J. (2008). Construct validity of the five-factor Strengths and Difficulties Questionnaire (SDQ) in pre-, early, and late adolescence. Journal of Child Psychology and Psychiatry, 49, 1304-1312.

Volbrecht, M., \& Goldsmith, H. (2010). Early temperamental and family predictors of shyness and anxiety. Developmental Psychology, 46, 1192 1205.

Werner, E., Myers, M., Fifer, W., Cheng, B., Fang, Y., Allen, R., \& Monk, C. (2007). Prenatal predictors of infant temperament. Developmental Psychobiology, 49, 474-484.

Zeanah, C. H., Boris, N. W., \& Larrieu, J. A. (1997). Infant development and developmental risk: A review of the past 10 years. Journal of the American Academy of Child \& Adolescent Psychiatry, 36, 165-178. 